\title{
Investigating and Promoting the Decision towards Signing an Organ Donation Card
}

\author{
Gundula Hübner ${ }^{1,2}$, Sonia Lippke ${ }^{3}$ \\ ${ }^{1}$ Medical School Hamburg, Hamburg, Germany \\ ${ }^{2}$ Martin-Luther-University Halle-Wittenberg, Halle, Germany \\ ${ }^{3}$ Jacobs University Bremen, Bremen, Germany \\ Email: gundula.huebner@psych.uni-halle.de, s.lippke@jacobs-university.de
}

Received 29 December 2013; revised 29 January 2014; accepted 6 February 2014

Copyright (C) 2014 by authors and Scientific Research Publishing Inc.

This work is licensed under the Creative Commons Attribution International License (CC BY). http://creativecommons.org/licenses/by/4.0/

(c) (i) Open Access

\begin{abstract}
Using the Health Action Process Approach (HAPA) as a theoretical backdrop, the aim of this research, which consists of two studies, was to understand the process of goal setting and deciding to sign an organ donor card. In Study $1(N=550)$, we tested the HAPA's stages in terms of discontinuity patterns in the variables predicting goal setting for organ donation. As expected, multigroup structural equation modeling revealed discontinuity patterns in terms of different prediction patterns. In Study $2(N=389)$, the efficacy of a planning intervention that aimed to foster the translation of goals into behavior was tested: participants who received the intervention were more likely to order a donor card than those who did not. Contrary to the HAPA's predictions, within the intenders group the planning intervention did not stimulate behavior more frequently than in the control condition. In general, the stage-specific planning intervention is of practical importance, as it shows that brief and relatively inexpensive communication can enhance organ donation behavior.
\end{abstract}

\section{Keywords}

Planning Intervention, Stage Model, Organ Donation, Goal Setting, Self-Efficacy

\section{Introduction}

Despite strong positive attitudes towards donating organs postmortem, and high intention to commit to such an act, only a few individuals actually sign an organ donor card. In order to promote organ donation i.e. preparatory behavior in terms of signing a donor card, it is important to understand the processes that drive this behavior. According to classical social-cognitive theories, the most immediate and important predictors of volitional, goal- 
directed behavior are goal intentions and plans [1] [2]. This raises the question: what determines the goal setting and translation of these goals into behavior in terms of plans? In the following, we outline the assumptions of a theoretical model and empirical evidence on explaining the behavior of signing an organ donation card.

\section{Theoretical Approach}

The Health Action Process Approach (HAPA, [3]) differentiates three stages of health behavior change. In the first (i.e. non-intentional) stage, in order to engage in a behavior, individuals must become highly motivated to make the decision to perform the behavior, and to set a goal (e.g., to become a potential organ donor and sign a donor card). The formation of the goal of performing the behavior (i.e. the intention) is determined by outcome expectancies (pros and cons like, for example, "organ donation helps to save lives" and "organ donors might not receive all possible medical treatment in the case of emergency", respectively), perceived self-efficacy (e.g., "I am capable of signing an organ donor card, even if my next of kin oppose organ donation”), and risk perception. Risk perception typically refers to a health risk that could be reduced by specific health behaviors (e.g., "I am at risk for cardiovascular disease." [4]). Risk perception, however, was found to be of less importance than the other social-cognitive variables [5].

If these variables lead an individual to set a goal (e.g., to sign a donor card), the intentional stage is entered. After the goal has been set (i.e. the intention is sufficiently high), positive and negative outcome expectancies become less important, while planning becomes crucial in translating the intentioninto behavior. Self-efficacy maintains its importance. As soon as an individual actually performs the behavior, the third stage (i.e. action stage) is reached and must be maintained. In this stage, self-efficacy is still imperative, as individuals always have to feel capable of performing the behavior [6] [7].

Previous observational studies and experiments provide evidence for the stages in terms of non-linearity across the HAPA stages, that is, different variables vary in their degree of importance depending on the stage. This is indicated by discontinuity in the variables' means and their interrelation across different stages [8] [9]). Non-linear, discontinuous pattern across stages can be represented by different means or predictions patterns [10].

As an example, a study on the maintenance of physical activity found significant differences in the average amount of self-efficacy present for behavior performance; it was lowest in the non-intentional stage, and highest in the action stage [11]. Additionally, intenders differed from non-intenders in terms of goals and plans, as well as correlation of negative outcome expectancies and intention. As expected, negative outcome expectancies showed a stronger correlation in the non-intentional stage than in the intentional stage.

However, a stronger test of stage assumptions is an experimentally test. Indeed, stage models have already been supported experimentally [e.g., 8]. An example of a previous experimental test in the exercise domain was a planning intervention, which was matched to the intentional stage, but mismatched to the non-intentional stage [8]. Support for the stage assumptions was found in terms of higher adherence rates in the planning intervention group only for study participants in the intentional stage, but not in the non-intentional stage.

While recent research suggests that the success of planning interventions differs, depending on which psychological stage people are in [8], stage-specific effects of a planning intervention addressing a single behavior — such as signing a donor card — have not been evaluated so far. Furthermore, in contrast to other health behaviors - such as regular physical exercise-postmortem organ donation and signing of organ donation cards are single behaviors that do not need to be maintained on a regular basis, and the individual does not benefit from the action itself. Moreover, to decide on postmortem organ donations requires the acceptance of one's own mortality - and thus might stimulate aversive emotions. So far it remains an open question as to whether the HAPA is a suitable model to explain this unique behavior, too.

Until now, the HAPA has been only partially tested in the domain of organ donation [12], in particular, people's communication of their consent for organ donation. Overall, research on promoting postmortem organ donation has indicated some effective methods. For example, persuasive messages and thought provocation successfully increased the general inclination to become a donor [13] [14], intention to sign a donor card [15] or motivation to seek further information about organ donation [16]. Additionally, persuasive attempts [13] [17] [18] were found to significantly improve the card signing rate. Simply stimulating an action plan of when to sign a donor card, however, resulted in a higher rate of card signing than persuasive communication [19]. This indicates that persuasive communications regarding organ donation may be unnecessary to encourage a person to become an organ donor. However, Birkimer's results [19] were probably confounded by a commitment effect, leading to higher signature rates. The procedure consisted of two sessions, with the second session being crucial 
for encouraging the decision: Participation in the second session was obligatory and the participants were informed that not deciding before the second session was equivalent to deciding against card signing. Therefore, it remains an open question as to whether a planning intervention introduced at a single meeting-a more applicable setting for practical communication campaigns-leads to comparably successful results. Generally, research on the concept of action plans and equivalent implementation intentions demonstrates promising results for successful planning interventions [2] [20]. The underlying assumption is that the more mental preparation is invested in an action, the higher the probability that an intended behavior will be initiated. Action plans are expected to improve cognitive links between situational circumstances or opportunities and goal behavior [20]; several studies in the health behavior domain have corroborated this assumption [21]-[23].

In sum, the aim of the present research is twofold. Firstly (in Study 1), we tested the stage-specific predictions of goal setting towards an altruistic, single behavior-donating an organ postmortem. The main innovation was to complement previous findings based on pure linear continuous assumptions in which the HAPA was chosen as a theoretical backdrop [12]. Subsequently (in Study 2), to validate the HAPA assumptions experimentally we tested the efficacy of a planning intervention introduced at a single meeting, and its stage-specific effects on the unique behavior of postmortem organ donation.

Study 1-Predicting goal setting to donate an organ: a test of the HAPA's assumptions

So far, only one stage theory, the Transtheoretical Model (TTM) [24], has been applied to analyze motivation to living donor kidney transplant [25], or motivation to agree to become a potential postmortem organ donor [26]. Keller and colleagues reported results comparable with those which are derived from the HAPA: individuals in the precontemplation stage, which is similar to the HAPA's non-intentional stage, showed lower self-efficacy in deciding to become a potential organ donor. Significantly, those in this precontemplation stage also perceived expected positive outcomes as less important and negative outcome expectancies as more important than individuals in other psychological stages. In this study, however, stage-specific relationships between continuous variables were not analyzed. Thus, it remains an open question as to whether the impact of variables predicting organ donation goal setting is moderated by psychological stages. Therefore, Study 1 of the current research tested the HAPA's ability to explain goal setting to become an organ donor.

In particular, this test involves three steps. First, stage assessment must be tested by examining differences between stages of groups in their means. According to the HAPA, we expect non-intenders and the further two stages (intenders and actors) to differ in their goal setting, with non-intenders reporting significantly less goal setting. Contrarily, intenders and actors are not expected differ from each other in terms of goal setting (hypothesis 1). Secondly, discontinuity patterns between means should demonstrate non-linear trends. If significant differences in means between all adjacent stages (e.g., non-intenders < intenders < actors) appeare in all test variables, this would support a linear continuous theory. Contrarily, a non-linear trend is indicated in general by significant differences between pairs of adjacent means in some variables, but not in others. Such a result pattern would support a stage model [12] [27]. Based on the HAPA's assumptions, mean discontinuity pattern hypotheses suggest that means of positive outcome expectancies should differ between the non-intentional and intentional stage (higher in intenders), but not between the intentional and action stage (hypothesis 2). Negative outcome expectancies should be highest in groups of non-intenders, but significant differences in these negative outcome expectancies should only exist between the non-intenders and intenders (hypothesis 3), not the intenders and actors. Furthermore, perceived self-efficacy is expected to be highest in the action stages, with significant differences between all three stages (hypothesis 4).

Third, in a structural equation analysis, the interrelation of the HAPA variables predicting donation goal setting negative outcome expectancies, positive outcome expectancies and self-efficacy-should be stage-dependent. We assume that non-intenders show higher interrelations of positive outcome expectancies and negative outcome expectancies with goals than people in other stages (hypothesis 5). In contrast, self-efficacy is expected to significantly correlate with goals in all stages, due to its generic function (hypothesis 6).

\subsection{Method}

\subsubsection{Sample and Procedure}

The sample consisted of 550 participants from a German middle sized town who were recruited primarily during university courses and leisure time meetings, such as choir rehearsals. An interviewer introduced the topic and asked individuals to answer the questionnaire. Due to voluntary participation, persons were allowed to leave the 
room, however, in all occasions the majority (about 94\%) of the people participated. Because organ donation is only viable for persons who do not reject it in principle, these participants were excluded from the analysis $(n=$ 32 ). Of the remaining 518 participants, all Caucasian, students were overrepresented (75.8\%), and they were enrolled in different majors (e.g., natural sciences: $24.4 \%$, economics: $23.4 \%$, social sciences: $22.5 \%$ ). Other participants came from heterogeneous backgrounds, such as school teachers or secretary staff, none of it represented by more than $4 \%$. Slightly more women (58.7\%) than men (41.3\%) participated. Ages ranged from 18 to 49 years; mean age was 22.53 years. The education level was comparatively high; the majority ( $88.6 \%)$ held a university entrance qualification.

On the front page of the questionnaire the topic was described, along with instructions on how to complete the questionnaire. The participants answered the questionnaires and handed them back to the conductors; participation was anonymous. A candy bar was offered as compensation for their time.

\subsubsection{Measures}

According to the HAPA, psychological stages are identified using the following criteria [7] [9]. Those participants who had neither signed an organ donor card, nor thought about it, were categorized as non-intenders. Participants who had thought about signing a card, but had a rather low intention (not answering absolutely agree to the question "I intend to sign a donor card") were also categorized as non-intenders. Those who had not signed a card, but had a high intention to do so (answering absolutely agree to the question "I intend to sign a donor card") were categorized as intenders. Participants who reported to have already signed a donor card, indicating their wish to donate, were grouped into the action stage.

To measure positive outcome expectations toward organ donation, we used a Likert-type scale with eight statements $(\alpha=0.81)$. The statements included the most frequent positive outcome expectancies related to organ donation, derived from existing organ donation research. This included questions like, for example, "organ donation helps to save lives" [28]. Participants were asked to rate on a six-point unipolar scale, how strongly they agreed with these statements, with responses ranging from 1 (do not agree at all) to 6 (absolutely agree).

Negative outcome expectations, were assessed by nine statements $(\alpha=0.89)$, such as "organ donors might not receive all possible medical treatment in case of emergency". This was also derived from existing organ donation research [28]. Participants were asked to rate on a six-point unipolar scale, how strongly they agreed with these statements, with responses ranging from 1 (do not agree at all) to 6 (absolutely agree).

Following the HAPA [8] [29], self-efficacy was composed of five items $(\alpha=0.91)$. Participants were asked to assess how certain they were that they could sign an organ donor card-thus, indicating their goals to donate-, even when facing obstacles. The five items followed the stem, "I am capable of signing an organ donor card even if...,” for example, “...my next of kin oppose organ donation.” Again, each item was rated on a six-point unipolar scale ranging from 1 to 6.

We assessed donation goals by four statements $(\alpha=0.90)$ referring to Horton and Horton's [30] research. Additionally, using this indicator increased comparability in the donation research domain. One item example is: “to what extent did you set the goal to donate organs after death?” Each item was rated on a six-point unipolar scale ranging from 1 to 6 , with the verbal anchors not at all and very.

\subsection{Data Analysis}

The HAPA and its discontinuity assumptions were tested in two steps. In the first step, the group differences in goal setting (hypothesis 1 ) and the mean discontinuity hypotheses (2-4) were tested with ANOVAs, and planned contrasts based on t-tests between adjacent stages. Secondly, the interrelation of the HAPA variables (hypotheses 5 and 6) was investigated by structural equation models, using the maximum likelihood method [31].

\section{Results}

\subsection{Distribution among the Three Stages}

The largest group in the sample, $60.2 \%(n=312)$, had neither signed an organ donor card, nor set a goal of doing so (non-intenders). About every fourth participant $(24.9 \% ; n=129)$ had already signed a donor card (actors). Only a minority $(14.9 \% ; n=77)$ reported that they had not signed a card, but had set the goal of doing so (intenders). 


\subsection{Group Differences in Goal Setting (Testing Hypothesis 1)}

Global differences between stage groups in their means in goals to donate were tested using an ANOVA. The overall effect was significant $F(2,515)=135.48, p<.001$, eta $^{2}=0.35$. As expected, post-hoc paired comparisons ( $t$-tests) provided evidence that non-intenders $(M=3.87, S D=1.21$ ) reported significantly (each $p<0.001)$ lower goalsthan the other groups (intenders: $M=5.17, S D=0.81$; actors: $M=5.50, S D=0.52$ ). However, contrary to expectations, the difference in means between intenders and actors was small but significant $(p<0.01)$.

\subsection{Stage-Specific Means of Social-Cognitive Variables (Testing Hypotheses 2, 3 and 4)}

To tackle differences between the stage groups, mean differences between the stages were analyzed. Separate ANOVAs for overall effects revealed that group means differed significantly between negative outcome expectancies $\left(F(2,515)=34.61, p<0.001\right.$ eta $\left.^{2}=0.12\right)$, self-efficacy $\left(F(2,515)=80.04, p<0.001\right.$, eta $\left.{ }^{2}=0.24\right)$ and positive outcome expectancies $\left(F(2,515)=2.99, p=0.05\right.$, eta $\left.^{2}=0.01\right)$.

Congruent with hypothesis 2 , paired comparisons revealed that non-intenders $(M=3.76, S D=0.93)$ reported significantly lower positive outcomes than intenders $(M=4.01, S D=0.95)$ and actors $(M=3.93, S D=0.87$; each $p<0.05$ ), while no significant difference was found between intenders and actors $(p<.60)$. Additionally, the data provided evidence for hypothesis 3: non-intenders $(M=3.02, S D=1.10)$ perceived significantly more negative outcome expectancies than intenders $(M=2.42, S D=0.82)$ and actors $(M=2.20, S D=0.82)$, each $p<$ 0.001 , while the difference between non-intenders and intenders was not significant $(p<0.10)$.

Furthermore, congruent with hypothesis 4, paired comparisons provided evidence that self-efficacy is significantly lowest in non-intenders $(M=4.04, S D=1.23)$, compared with intenders $(M=5.21, S D=0.71)$ and actors $(M=5.26, S D=0.72$; each $p<0.001)$. However, contrary to the same hypothesis, the difference between intenders and actors was not significant $(p<0.40)$. In summary, discontinuity patterns in terms of nonlinear trends were found in all of the three test variables (significant differences between non-intenders and intenders, and no mean differences between intenders and actors). All but two predicted mean differences were found in the expected manner.

\subsection{Testing the HAPA across the Three Stage Groups}

In a first step, the postulated HAPA structure was tested using structural equation analyses (with AMOS) for each group separately. The hypothesized model could be applied to a multigroup comparison only if it had an acceptable model fit for all stage groups separately [32]. The fit statistics indicated that the model representing the HAPA could be accepted from an empirical point of view. The model fit indicators, which are comparatively insensitive to sample size (i.e. comparative-fit-index, CFI, and root-mean-square error of approximation, RMSEA), suggested that the model fit was acceptable for the three groups, with a relatively low error of approximation in the non-intentional $(\mathrm{CFI}=0.99$, RMSEA $=0.03)$ and action stages $(\mathrm{CFI}=0.98, \mathrm{RMSEA}=0.06)$, but with a reasonable error of approximation in the intentional stage $(\mathrm{CFI}=0.94$, RMSEA $=0.11)$. Since the fit statistics indicated a reasonable fit in the three groups overall, the moderating role of psychological stages was tested by simultaneous group comparisons using multigroup structural equation modeling.

\subsection{Testing the Moderating Role of Psychological Stages (Hypotheses 5 and 6)}

To determine whether the measurement models of theoretical constructs operated equivalently across stage groups, a sequence of nested models ranging from an unconstrained multi-group model with freely estimated parameters to more parsimonious models including different levels of equality constraints, was first calculated [31] [32]. Fit statistics for these different models are summarized in Table 1.

Model 1 represents the unconstrained model, which was compared to more constrained models. To test for measurement equivalent models across the three subgroups, equal factor loadings across subgroups were specified in Model 2. Models 1 and 2 provided good model fits (Table 1). More importantly, the $\chi^{2}$ difference between the models was not significant, confirming an invariant measurement model for the three stage groups. Next, in addition to factor loadings, covariances were fixed as equal across the stage groups (Model 3). Again, Model 3 provided a good model fit, and did not differ significantly from the unconstrained Model 1 . Thus, Model 3 accounted for the subgroup's covariances, as well as Model 1. Further, in Model 4, factor variances were constrained. However, this model proved to be significantly different from Model 1, suggesting that factor 
Table 1. Three group nested models and $\chi^{2}$ differences with increased constraints.

\begin{tabular}{rccccccccc}
\hline Model & $\chi^{2}$ & $d f$ & $\chi^{2} / d f$ & $p$ & CFI & RMSEA & $\begin{array}{c}\text { Model 1 } \\
\Delta \chi^{2}\end{array}$ & $\begin{array}{c}\text { Model 1 } \\
p\end{array}$ \\
\hline Model 1 & 98.0 & 63 & 1.56 & $<0.01$ & 0.98 & 0.03 & - & - \\
Model 2 & 100.1 & 69 & 1.45 & $<0.01$ & 0.99 & 0.03 & 2.1 & $<0.95$ \\
Model 3 & 110.3 & 75 & 1.47 & $<0.001$ & 0.98 & 0.03 & 12.3 & $<0.50$ \\
Model 4 & 163.7 & 81 & 2.02 & $<0.001$ & 0.96 & 0.05 & 65.7 & $<0.001$ \\
Model 5 & 135.9 & 81 & 1.68 & $<0.001$ & 0.98 & 0.04 & 37.9 & $<0.05$ \\
\hline
\end{tabular}

Note: Model 1, unconstrained model; Model 2, measurement equivalent models (equal factor loadings across the subgroups). Model 3, additionally to Model 2's constrains covariances equivalence models. Model 4, additionally to Model 3's constrains the factor variances were constrained; Model 5, Model 3's constraints plus equal paths.

variances were mostly sample-specific.

The most parsimonious model (i.e. Model 3), which varied non-significantly from the unrestricted Model 1 , was chosen to test the relationships of the model's variables across subgroups [31]. To test whether the causal structure implied by the HAPA was unique across stages, Model 3 constraints (constrained factor loadings and factor covariances), plus equal paths across subgroups, were specified in Model 5. Congruent with expectations, Model 5 appeared to be significantly different from Model 1, thus providing evidence for mostly subgroup-specific paths. From a statistical point of view, Model 3, which allows for unique paths between stages, was the best representation of the data.

The interrelations between positive and negative outcome expectancies were almost zero across subgroups (non-intenders $-0.01 /$ intenders -0.02 /actors -0.02). All other HAPA determinants were correlated below 0.50 (interrelation of negative outcome expectancies and self-efficacy: non-intenders -0.19 /intenders $-0.45 /$ actors -0.49; interrelation of positive outcome expectancies and self-efficacy: 0.10/0.20/0.24).

Figure 1 shows the factors' association with goals in the three stage groups. Across groups, negative outcome expectancies and self-efficacy had a significant intercorrelation with goals to become an organ donor, while expected outcomes were only significant for non-intenders. Congruent with hypothesis 5 , subsequent pairwise parameter comparisons showed that the impact of positive outcome expectancies was significantly stronger for the non-intenders than for the intenders and actors.

In addition, hypothesis 5, concerning the intercorrelation of negative outcome expectancies with goals, was confirmed: negative outcome expectancies had a significantly stronger correlation with goals in non-intenders than in intenders. However, contrary to our assumptions, no differences were observed between non-intenders and actors. Testing hypothesis 6, self-efficacy appeared to have the strongest intercorrelation in the group of intenders, while non-intenders and actors did not differ significantly from each other. Thus, hypothesis 6 was confirmed: self-efficacy was significantly interrelated with goals in all stages, proving its generic function, and significant differences between stage groups indicated further discontinuity patterns.

Finally, explained variances differed between the three subgroups, providing further evidence for discontinuity patterns between the stages. For non-intenders, about $51 \%$ of the variance in goalswas explained, for intenders $77 \%$ and for actors $64 \%$.

\section{Discussion}

The results of Study 1 of the study provide evidence for the proposed structure of goal setting within the HAPA framework. Goal setting was operationalized in terms of committing to postmortem organ donation. Most hypotheses on mean comparison were confirmed, although only one difference between actors and intenders was found. Contrary to our expectations, goals were different not only between non-intenders and intenders, but also between intenders and actors. This was also found in a study testing the HAPA in the physical activity domain [11]. Additionally, as expected, the average mean of expected outcomes was lower in non-intenders than in intenders and actors. This matches the findings of Keller et al. [26], that non-intenders expected less positive outcomes than other stage groups.

The interrelation of the positive outcome expectancies and negative outcome expectancies, as well as self-efficacy regarding donation goals, differed significantly between the three stage groups. More specifically, only non-intenders showed significant interrelations of positive outcome expectancies with goals. Negative outcome 


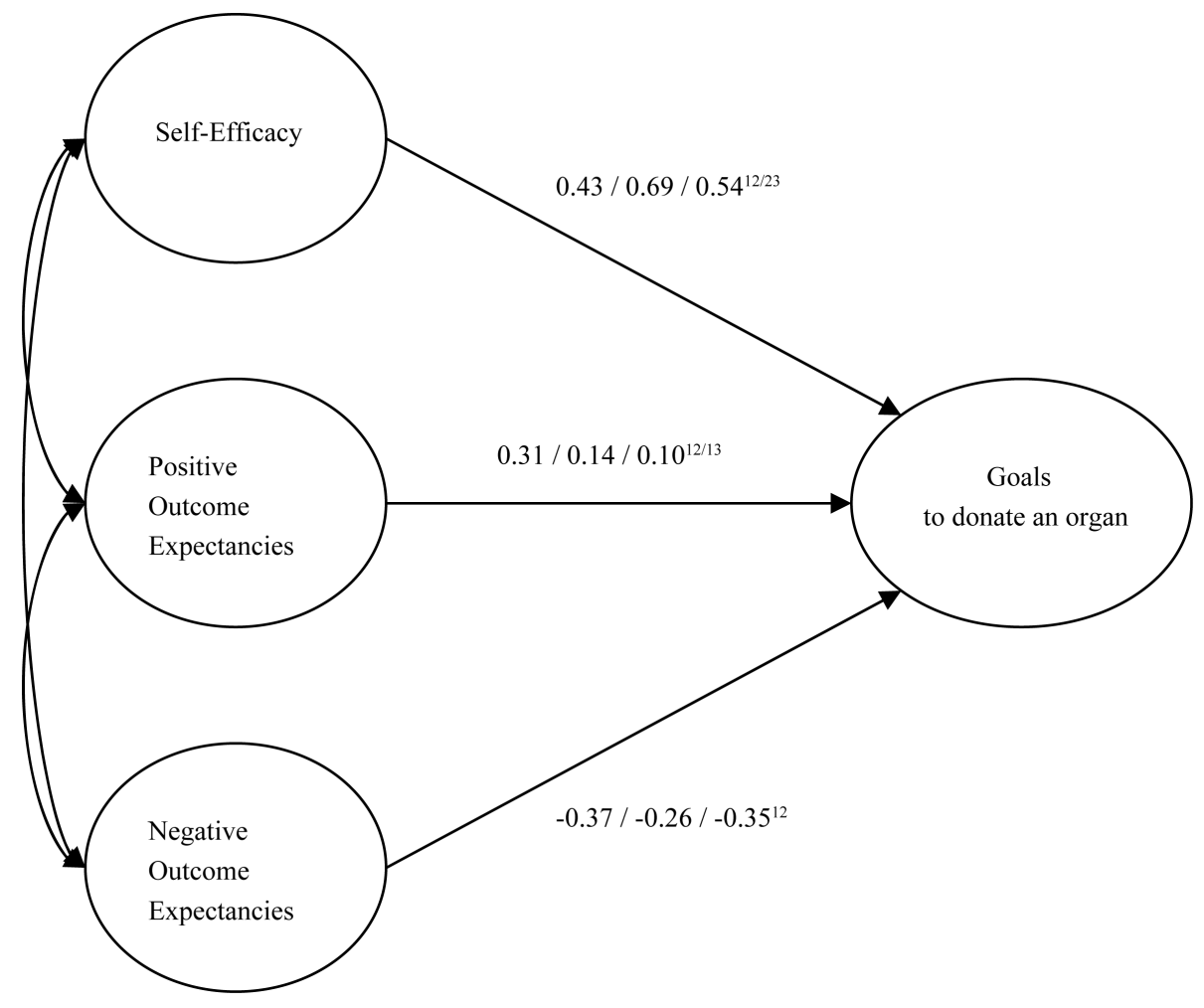

Figure 1. Standardized regression coefficients for the measurement equivalence model (Model 3) across the three stage subgroups. Note: Significant coefficients are in bold; truncated numbers indicate significant differences of the regression coefficients between subgroups: " 12 " = non-intenders and intenders differ significantly, " 13 " = non-intenders and actors, and " 23 " = intenders and actors. Values are listed for the theoretically prominent parameters only, but not for the measurement model.

expectancies and self-efficacy were interrelated with goals in all three groups. Contrary to our assumptions, the intercorrelation of negative outcome expectancies with goals was comparatively high in non-intentional and action stages. However, the average amount of negative outcome expectancies was highest in the non-intender group. For the prediction of donation goals, self-efficacy seemed to be the most important variable when the decision to sign a donor card had been made, but had not yet been implemented.

One shortcoming of the present study is that in the unique case of postmortem organ donation, it is impossible to observe the actual donation or a directly connected volitional phase. Instead, to define the stages we had to switch to a preparatory behavior. Consequently, we cannot claim to have validated the HAPA in its complexity. Rather, one might argue that we stayed within the motivational stage concerning organ donation. However, even in this special case, we found the expected stage specific discontinuity patterns suggested by the HAPA. Further, Study 1 is limited by its cross-sectional, correlational research design, and its sole reliance on self-reports. Furthermore, it includes past behavior (in terms of the stage variable), but no future behavior, no planning and no experimental manipulation. An experimental test is imperative for testing stages [12]. Thus, in the subsequent Study 2, the HAPA assumptions were tested experimentally, by adding a planning intervention and a preparatory behavior measure.

Study 2-Testing a Volitional Planning Intervention Promoting the Decision towards the Signing of an Organ Donation Card

The first goal of Study 2 was to overcome the previous study's shortcoming of the cross-sectional design and to experimentally test the HAPA's validity to explain organ donation behavior. Former research on planning interventions confirmed the existence of specific behavior stages [8] [12] [33], for an overview, see [10]. As mentioned, a first study on the effects of a planning intervention to stimulate organ donation behavior [19] included two measurement times that likely stimulated an additional public commitment effect. It remains an open question as to whether a single point intervention can be effective to stimulate a single-act behavior, such as signing 
an organ donor card. Therefore, the second goal of Study 2 was to examine the impact of a single intervention on a proximal behavior measure (preparatory behavioral).

In other studies, a planning intervention appeared to be optimal for people who have already decided to initiate a new action (intentional stage). By contrast, individuals in the non-intentional stage first have to set a goal (i.e. increase their goals to donate) [8] [12]. For this group, a pure planning intervention is mismatched. The second purpose of Study 2 was therefore to investigate these stage-specific effects of a planning intervention, designed to translate goals to donate organs postmortem, into actually signing a donor card. Specifically, we expected individuals in the intentional stage to profit more from the planning intervention than those in the nonintentional stage.

\subsection{Method}

\subsubsection{Sample and Procedure}

The participants were a subset of the sample from Study 1 . Those who already possessed an organ donor card were excluded from analyses of Study 2. Hence, the remaining sample size was $N=389$. After participants answered the items used to measure HAPA constructs in Study 1, the planning intervention followed in the last part of the same questionnaire.

Participants were unsystematically assigned to one of two experimental conditions. More specifically, the interviewers distributed the questionnaires, including the planning intervention, without a specific order. In the intervention condition, participants answered the questionnaire, which included a short planning intervention. In the control condition, participants answered the questionnaire only. None of the study participants dropped out from the study.

For the analyses, individuals in both the control group and the intervention group were subdivided according to their stage: $n=125$ participants in the non-intentional stage were in the control group, and $n=187$ in the intervention group. Preliminary analyses that were conducted to determine the comparability of these two groups revealed no differences in terms of mean age and gender (each $p>0.68$ ). Furthermore, no significant differences were obtained in the HAPA variables (each $p>0.19$ ). Of those in intentional stage, $n=27$ were in the control condition, and $n=50$ received the intervention. Again, these two groups did not differ significantly in terms of age, gender (each $p>0.24$ ) and HAPA variables (all $p$ s $>0.23$ ).

\subsubsection{Planning Intervention}

The participants were first informed about where they could get an organ donor card and how to sign it. Next, the participants were encouraged to form an action plan by specifying where they planned to get a card, when they planned to sign it, and where they planned to keep it. In addition, they were asked to write down their plan on a planning sheet.

\subsection{Measures}

All measures were identical to those described in Study 1, except that a behavior measure was added. Preparatory Behavior was operationalized as either requesting an organ donor card after the survey, or not requesting one. A postcard was included on the second-to-last page of the questionnaire. The card was attached to the questionnaire; postcard and questionnaire had identical numbers. Participants could exchange the postcard for an organ donor card when returning the questionnaire. This operationalization was chosen to make it easy and convenient for participants to get an organ donor card, and to actually measure one behavioral indicator within the study.

\subsection{Results}

To test effects of stages and the planning intervention on behavior, a binary logistic regression was computed. Results, summarized in Table 2, revealed a significant main effect for the planning intervention, as well as for stage. The significant main effect of the planning intervention indicated that this intervention resulted in more preparatory behavior, as compared to the control group. In the intervention group, $24.1 \%$ of the participants requested a donor card, compared to $17.8 \%$ in the control group. Taken together, the predicted odds suggest that an individual is about nine times as likely to sign a card if he or she has set the goal to become an organ donor, and about twice as likely to do so if he or she received a planning intervention—independent of stage (cf. Table 2). 
Table 2. Results of the logistic regression predicting card requesting behavior with stage, planning intervention and their interaction.

\begin{tabular}{cccccc}
\hline Variable & B & SE B & OR & Wald statistic & $p$ \\
Stage & 2.23 & 0.48 & 9.28 & 21.19 & 3.88 \\
Planning intervention & 0.68 & 0.35 & 1.98 & 0.001 & 0.05 \\
Stage * Planning intervention & -1.00 & 0.59 & 0.37 & 2.86 \\
\hline
\end{tabular}

Note: $\mathrm{N}=389$; Nagelkerke $\mathrm{R}^{2}=0.14$.

Participants in the intentional stage more frequently requested an organ donor card than participants in nonintentional stage; $46.8 \%$ versus $15.4 \%$; $p<0.001$. A marginally significant interaction between stage and intervention provided evidence for a stage-specific effect of the planning intervention. However, the interaction was opposite to the hypothesis: the planning intervention seemed to be more successful in the non-intentional stage, in which more participants in the intervention group requested a card, compared with the control group (cf. Figure 2). A reverse result pattern appeared in the intentional stage. For intenders, the frequency of requested cards was higher in the control group than in the intervention group. Together, the main effects and the interaction term explained $14 \%$ of the behavioral variation.

\subsection{Discussion}

Participants who had been encouraged to form an action plan requested a donor card about twice as often as participants who had only answered the questionnaire. Apparently, a structured opportunity to request and sign an organ donor card was effective in improving behavior. As expected, data alluded to a stage-specific impact of the planning intervention. However, this effect was only marginally significant and importantly, opposite to expectations and former research [8] [12]: For non-intenders, the planning interventions stimulated relatively more card request compared to the control condition. However, within intenders, the planning intervention appeared to be less efficient than simply offering the donor card. A possible explanation for this unexpected poorer performance of the planning intervention could be that signing an organ donor card is a single, easy-to-execute preparatory behavior, which does not require much further self-regulation. Most likely, in this specific case, the crucial point is making the decision and translating it into actual action, namely signing an organ donor card. If the goal was actually set, this seems to require no further preparation or action plan. This assumption is underlined by the finding that only a minority of our sample were intenders. Almost half of the intenders took the opportunity to get an organ donor card. This behavioral rate was lower in non-intenders, providing evidence for the HAPA assumption that intenders are closer to the action stage.

We did not, however, assess whether the participants had already received a donor card but just had not signed it, or whether they knew an alternative way of easily obtaining one (e.g., at the pharmacy or the Internet). All of these individuals would form a sub-group of the typical intenders, and not get the donor card at the study site, but instead sign the already existing card or get one at another place, respectively. The actual behavior investigated in this study was a small step; a stronger behavioral measure would be to control for the actual signature behavior. Nevertheless, requesting an organ donor card is a potentially important step that suggests some movement in the direction of actually becoming an organ donor.

\section{General Discussion}

To our knowledge, this study is the first research on the basic theory of stage-specific interrelations of socialcognitive variables, and the impact of a brief action planning intervention. The study has yielded a number of important findings. Firstly, the HAPA, with its variables and stages, appears to be a promising approach to understanding a person's decision to become a postmortem organ donor. Study 1 provided evidence for discontinuity patterns across the stages. Congruent with the HAPA's assumptions, non-intenders expected significantly less positive outcomes from donating organs, were less self-assured about signing a donor card, and held negative outcome expectancies to a higher degree than intenders. Interestingly, intenders and actors on average showed no significant differences. This finding corroborates earlier results in the organ donation domain, providing evidence that it is not the extent of belief in positive aspects of organ donation that differentiates between donors and non-donors, but rather the negative outcome expectancies or negative emotions associated with or- 


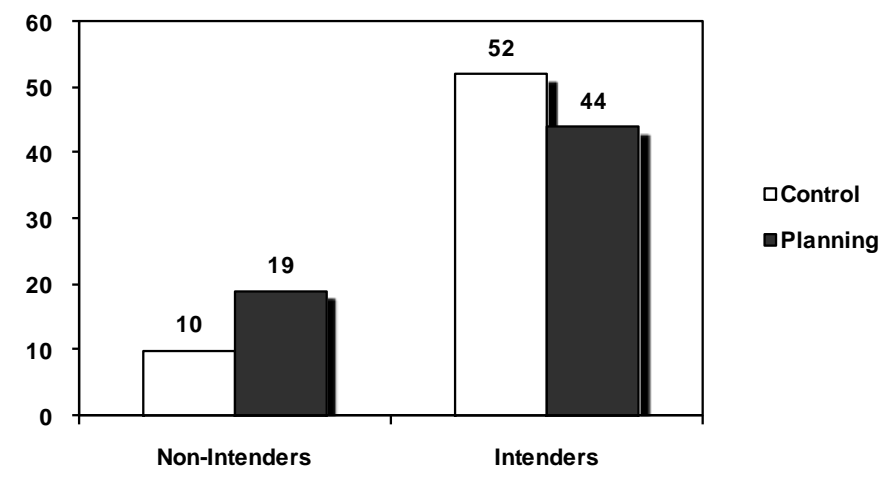

Figure 2. Requested cards by stage and planning (in percent).

gan donation [34].

More importantly, the data of the present research reveal that the intercorrelation of the presented social-cognitive variables with goals is moderated by stages specified in the HAPA. That is, in the non-intentional stage, self-efficacy, positive outcome expectations, and negative outcome expectancies are interrelated with donation goals. The moment people commit themselves to signing a donor card, only negative outcome expectancies accompanied by self-efficacy intercorrelate with donation goals. This indicates discontinuity patterns and non-linearity. The same holds true for positive outcome expectancies, which are significantly related to goals in nonintenders, but not in intenders and actors. Furthermore, the mean structure and the interrelation of the variables across the stages indicate a discontinuity pattern. This finding supports the HAPA stages in particular, and stage assumptions in general [10].

The finding that self-efficacy appeared to be more important than planning in the intentional stage might be due to the fact that the decision to accept postmortem organ donation is a single behavior. Contrary to behaviors such as regular physical exercise [11], goals to donate organs after death do not need to be maintained, so self-efficacy is of crucial importance at the moment of decision-making. Moreover, organ donation is a very unique behavior - it is a single action and involves offering parts of one's own body. It is very likely related to different cognitions and emotions, as compared to other behaviors. If so, our results might suggest that planning interventions should be more behavior specific. Since the present research is the first to apply the HAPA to a single behavior such as signing an organ donor card, further evidence is needed to prove this assumption — even more so since we assessed the action stage retrospectively and by self-report, and did not have the chance to assess the volitional phase adequately. Despite these shortcomings, our findings are in line with studies that have validated the HAPA's basic assumptions [4].

The second aim of our research was to test the impact of a planning intervention designed to stimulate a behavior, in this study, committing oneself to become an organ donor after death. Our results corroborate previous findings [12] [19] that merely encouraging the decision by stimulating a behavior plan can be an effective intervention to increase organ donation behavior. This type of intervention would at least be useful for single behaviors. More importantly, contrary to Birkimer and colleagues [12], our intervention included only one intervening point in time instead of two, in order to come from a more practical point of view. However, Birkimer's study differed in that it compared effects of a planning intervention with a message based only on factual information. Thus, in this study it remains open as to whether combining a planning intervention with persuasive communication, simultaneously incorporating empathy and knowledge, would increase planning.

Contrary to expectations, the planning intervention seemed marginally less successful within the intentional stage: intenders who received the planning instruction requested fewer cards than those who received no instruction. A possible explanation for this unexpected negative interaction could be the behavior's simplicity: the planning instruction might have evoked resistance in some participants. An alternative explanation could be that those intenders who were asked to do a planning intervention may have felt less willing to turn in their postcard and get a donor card right away in comparison to those not asked to plan. Even the clear decision to sign seems to be a necessary, but not sufficient condition for subsequent behavior, as was found to be typical in various behaviors [2] [20]. A possible explanation for this finding could be that the behavior was measured immediately after the intervention. Typically, planning works if planned components (such as when, where and how) appear 
at a later point in time [2] [7]. For this reason, it could not be detected whether some intenders chose to request a donor card from alternative sources, such as via the Internet. Another explanation would be that participants indicated higher levels of intention to sign a donor card due to social desirability. However, since the study was conducted anonymously this explanation seems unlikely.

Interestingly, some non-intenders in the intervention proceeded to the action stage: more non-intenders who received a planning intervention (which was designed as a mismatched intervention) changed their behavior than those who did not receive such an intervention. Those who were in the non-intentional stage were probably encouraged by the planning intervention to realize how few obstacles there actually are to becoming an organ donor, and how easy signing a card can be. An additional possibility is that these participants, who had not thought about becoming an organ donor before, took the card out of curiosity, or to get further information. However, this is again speculation, and further research is needed to understand the underlying mechanisms of this result.

Our research is the first to test the efficacy of a stage-specific planning intervention to promote signing an organ donor card. The results are promising, and replicate findings in other behavioral domains. However, more research is needed to test add-on effects of a planning intervention to persuasive communication, or other stagematched interventions [33]. As well, future studies should explicitly investigate the goal setting towards signing a donor card, instead of assessing intentions towards donating organs post mortem. In conclusion, discontinuity patterns observed in variables predicting donation goals and the planning intervention provide further evidence support for the HAPA. Moreover, the results are of practical importance, as they show that brief and relatively inexpensive communication can enhance organ donation preparatory behavior.

\section{References}

[1] Ajzen, I. and Fishbein, M. (2005) The Influence of Attitudes on Behavior. In: Albarracin, D., Johnson, B.T. and Zanna, M.P., Eds., The Handbook of Attitudes, Erlbaum, Mahwah, 173-221.

[2] Gollwitzer, P.M. and Sheeran, P. (2006) Implementation Intentions and Goal Achievement: A Meta-Analysis of Effects and Processes. Advances in Experimental Social Psychology, 38, 69-119. http://dx.doi.org/10.1016/S0065-2601(06)38002-1

[3] Schwarzer, R. (2008) Modeling Health Behavior Change: How to Predict and Modify the Adoption and Maintenance of Health Behaviors. Applied Psychology: An International Review, 57, 1-29. http://dx.doi.org/10.1111/j.1464-0597.2007.00325.x

[4] Schwarzer, R., Lippke, S. and Luszczynska, A. (2011) Mechanisms of Health Behavior Change in Persons with Chronic Illness or Disability: The Health Action Process Approach (HAPA). Rehabilitation Psychology, 56, 161-170. http://dx.doi.org/10.1037/a0024509

[5] Milne, S., Sheeran, P. and Orbell, S. (2000) Prediction and Intervention in Health-Related Behavior: A Meta-Analytic Review of Protection Motivation Theory. Journal of Applied Social Psychology, 30, 106-143. http://dx.doi.org/10.1111/j.1559-1816.2000.tb02308.x

[6] Schwarzer, R. (1992) Self-Efficacy in the Adoption and Maintenance of Health Behaviors: Theoretical Approaches and a New Model. In: Schwarzer, R., Ed., Self-Efficacy: Thought Control of Action, Hemisphere, Washington DC, 217243.

[7] Schwarzer, R. (2001) Social-Cognitive Factors in Changing Health-Related Behaviors. CurrentDirections in Psychological Science, 10, 47-51. http://dx.doi.org/10.1111/1467-8721.00112

[8] Lippke, S., Ziegelmann, J.P. and Schwarzer, R. (2004) Initiation and Maintenance of Physical Exercise: Stage-Specific Effects of a Planning Intervention. Research in Sports Medicine, 12, 221-240. http://dx.doi.org/10.1080/15438620490497567

[9] Lippke, S., Ziegelmann, J.P., Schwarzer, R. and Velicer, W.F. (2009) Validity of Stage Assessment in the Adoption and Maintenance of Physical Activity and Fruit and Vegetable Consumption. Health Psychology, 28, 183-193. http://dx.doi.org/10.1037/a0012983

[10] Sutton, S. (2005) Stage Theories of Health Behavior. In: Conner, M. and Norman, P., Eds., Predicting Health Behavior, Open University Press, Maidenhead, 223-275.

[11] Lippke, S., Ziegelmann, J.P. and Schwarzer, R. (2005) Stage-Specific Adoption and Maintenance of Physical Activity: Testing a Three-Stage Model. Psychology of Sport and Exercise, 6, 585-603. http://dx.doi.org/10.1016/j.psychsport.2004.11.002

[12] Hydeabc, M.K. and Whitebc, K.M. (2013) A Test of Three Interventions to Promote People’s Communication of Their Consent for Organ Donation. Psychology \& Health, 28, 399-417. http://dx.doi.org/10.1080/08870446.2012.731060 
[13] Morgan, S.E., Miller, J. and Arasaratnam, L.A. (2002) Signing Cards, Saving Lives: An Evaluation of the Worksite Organ Donation Promotion Project. Communication Monographs, 69, 253-273. http://dx.doi.org/10.1080/03637750216540

[14] Singh, M., Katz, R.C., Beauchamp, K. and Hannon, R. (2002) Effects of Anonymous Information about Potential Organ Transplant Recipients on Attitudes toward Organ Transplantation and the Willingness to Donate Organs. Journal of Behavioral Medicine, 25, 469-476. http://dx.doi.org/10.1023/A:1020471007837

[15] Skumanich, S.A. and Kintsfather, D.P. (1996) Promoting the Organ Donor Card: A Causal Model of Persuasion Effects. Social Science and Medicine, 43, 401-408. http://dx.doi.org/10.1016/0277-9536(95)00404-1

[16] Ford, L.A. and Smith, S.W. (1991) Memorability and Persuasiveness of Organ Donation Message Strategies. American Behavioral Scientist, 34, 695-711. http://dx.doi.org/10.1177/0002764291034006007

[17] Sanner, M.A., Hedman, H. and Tufveson, G. (1995) Evaluation of an Organ Donor Card Campaign in Sweden. Clinical Transplantation, 9, 326-333.

[18] Skowronski, J.J. (1990) Increasing the Number of People Who Agree to Donate Organs: Can Persuasion Work? In: Shanteau, J. and Harris, R.J., Eds., Organ Donation and Transplantation: Psychological and Behavioural Factors, American Psychological Association, Washington DC, 122-135.

[19] Birkimer, J.C., Barbee, A.P., Francis, M.L., Berry, M.M., Deuser, P.S. and Pope, J.R. (1994) Effects of Refutational Messages, Thought Provocation, and Decision Deadlines on Signing to Donate Organs. Journal of Applied Social Psychology, 24, 1735-1761. http://dx.doi.org/10.1111/j.1559-1816.1994.tb01572.x

[20] Bélanger-Gravela, A., Godin, G. and Amireault, S. (2013) A Meta-Analytic Review of the Effect of Implementation Intentions on Physical Activity. Health Psychology Review, 7, 23-54. http://dx.doi.org/10.1080/17437199.2011.560095

[21] Armitage, C.J. (2004) Evidence that Implementation Intentions Reduce Dietary Fat Intake: A Randomized Trial. Health Psychology, 23, 319-323. http://dx.doi.org/10.1037/0278-6133.23.3.319

[22] Rocheleau, C.A. (2013) Organ Donation Intentions and Behaviors: Application and Extension of the Theory of Planned Behavior. Journal of Applied Social Psychology, 43, 201-213. http://dx.doi.org/10.1111/j.1559-1816.2012.00998.x

[23] Sheeran, P. and Orbell, S. (2000) Using Implementation Intentions to Increase Attendance for Cervical Cancer Screening. Health Psychology, 19, 283-289. http://dx.doi.org/10.1037/0278-6133.19.3.283

[24] Prochaska, J.O. and Velicer, W.F. (1997) The Transtheoretical Model of Health Behavior Change. American Journal of Health Promotion, 12, 38-48. http://dx.doi.org/10.4278/0890-1171-12.1.38

[25] Waterman, A.D., Robbins, M.L., Paiva, A.L., Peipert, J.D., Davis, L.A., Hyland, S.S., Schenk, E.A., Baldwin, K.A. and Amoyal, N.R. (2014) Measuring Kidney Patients’ Motivation to Pursue Living Donor Kidney Transplant: Development of Stage of Change, Decisional Balance and Self-Efficacy Measures. Journal of Health Psychology, in press.

[26] Keller, S., Bölting, K., Kaluza, G., Schulz, K.H., Ewers, H., Robbins, M.L. and Basler, H.D. (2004) Bedingungen für die Bereitschaft zur Organspende [Prerequisites for the Resolve to Become an Organdonor]. Zeitschrift für Gesundheitspsychologie, 12, 75-84. http://dx.doi.org/10.1026/0943-8149.12.2.75

[27] Sutton, S. (2000) Interpreting Cross-Sectional Data on Stages of Change. Psychology \& Health, 15, $163-171$. http://dx.doi.org/10.1080/08870440008400298

[28] Radecki, C.M. and Jaccard, J. (1997) Psychological Aspects of Organ Donation: A Critical Review and Synthesis of Individual and Next-of-Kin Donation Decisions. Health Psychology, 16, 183-195. http://dx.doi.org/10.1037/0278-6133.16.2.183

[29] Lippke, S. and Plotnikoff, R.C. (2014) Testing Two Principles of the Health Action Process Approach in Individuals with Type 2 Diabetes. Health Psychology, 33, 77-84.

[30] Horton, R.L. and Horton, P.J. (1991) A Model Willingness to Become a Potential Organ Donor. Social Science and Medicine, 33, 1037-1051. http://dx.doi.org/10.1016/0277-9536(91)90009-2

[31] Lippke, S., Nigg, C.R. and Maddock, J.E. (2007) The Theory of Planned Behavior within the Stages of the Transtheoretical Model: Latent Structural Modeling of Stage-Specific Prediction Patterns in Physical Activity. Structural Equation Modeling: A Multidisciplinary Journal, 14, 649-670. http://dx.doi.org/10.1080/10705510701575586

[32] Byrne, B.M. (2001) Structural Equation Modeling with Amos: Basic Concepts, Applications, and Programming. Erlbaum, Mahwah.

[33] Blissmer, B. and McAuley, E. (2002) Testing the Requirements of Stages of Physical Activity among Adults: The Comparative Effectiveness of Stage-Matched, Mismatched, Standard-Care, and Control Interventions. Annals of Behavioral Medicine, 24, 181-189. http://dx.doi.org/10.1207/S15324796ABM2403_03

[34] Parisi, N. and Katz, L. (1986) Attitude towards Posthumous Organ Donation and Commitment to Donate. Health Psychology, 5, 565-580. http://dx.doi.org/10.1037/0278-6133.5.6.565 


\section{Appendix Item Examples}

Positive Outcome Expectations Subscale.

Organ donation helps to save lives.

Organ donation gives some sense to death.

A person willing to donate an organ postmortem lives more consciously.

Donating an organ enables that part to remain alive after a person's death.

Negative Outcome Expectations Subscale.

Potential organ donors might not receive all possible medical treatment in case of emergency.

Organ donation leaves the body mutilated.

In the case of organ donation, the body may be treated disrespectfully.

Organ donation disturbs the peace of the deceased.

\section{Self-Efficacy:}

I am capable of signing an organ donor card even if...

... my next of kin oppose organ donation.

... my friends do not like my decision.

... others try to prevent me from my decision.

... my goal will be questioned.

... I do not have much time to decide.

\section{Donation Goals}

To what extent did you set the goal to donate organs after death?

How willing are you to donate organs after death?

How much to you intend to communicate to your next of kin your decision to donate.

To what extent have you set the goal to sign a donor card? 